\title{
Influência de Variáveis Meteorológicas na Prevalência das Doenças Transmitidas pelo Mosquito Aedes Aegypti
}

\author{
Roberto Alan Ferreira Araújo $^{1}$ (D), Natália Macêdo Uchôa ${ }^{2}$ (D), José Maria Brabo Alves ${ }^{2}$ \\ ${ }^{I}$ Departamento de Engenharia Civil, Centro Universitário, Faculdades Integradas de Patos, \\ Patos, PB, Brasil. \\ ${ }^{2}$ Mestrado Profissional em Climatologia e Aplicações nos Países da CPLP e África, \\ Centro de Ciências e Tecnologia, Universidade Estadual do Ceará, Fortaleza, CE, Brasil.
}

Recebido em: 30 de Abril de 2019 - Aceito em: 14 de Julho de 2019

\begin{abstract}
Resumo
Atualmente as pessoas têm relacionado problemas de saúde às mudanças climáticas de forma direta ou indireta, ocasionando o aumento da prevalência de certas doenças. O trabalho mostra como as variáveis meteorológicas influenciam na prevalência das doenças transmitidas pelo mosquito Aedes aegypti. Foram analisados dados coletados Sistemas de Monitoramento Diário de Agravos (SIMDA) da Secretaria de Saúde do Município de Fortaleza e os dados meteorológicos foram coletados do site Instituto Nacionais de Meteorologia (INMET) referente ao período de Janeiro de 2013 a Dezembro de 2017. Para verificar as influências dos elementos climáticos no desencadeamento da Dengue, Chikungunya e Zika o presente trabalho buscou relacionar as variáveis climáticas com as doenças por estações do ano e por semestre.
\end{abstract}

Palavras-chave: variáveis climáticas, Aedes aegypti, dengue, Chikungunya, Zika.

\section{Influence of Meteorological Variables on the Prevalence of Diseases Transmitted by Aedes Aegypti Vector}

\begin{abstract}
People today have directly or indirectly related health problems to climate change, causing an increase in the prevalence of certain diseases. The work shows how the meteorological variables influence the prevalence of diseases transmitted by the Aedes aegypti mosquito. Data were collected from the Daily Health Monitoring Systems (SIMDA) of the Health Department of the Municipality of Fortaleza and the meteorological data were collected from the National Institute of Meteorology (INMET) website for the period from January 2013 to December 2017. To verify the influence of the climatic elements in the triggering of Dengue, Chikungunya and Zika the present work sought to relate the climatic variables to the diseases by seasons and by semester.
\end{abstract}

Keywords: climate variables, Aedes aegypti, dengue, Chikungunya, Zika.

\section{Introdução}

Não é novidade que a variabilidade climática está produzindo efeitos diretos e indiretos na saúde humana, entretanto nunca se deu tanta atenção às pesquisas que envolvem os temas estudados pela Biometeorologia como atualmente. Isto é devido aos resultados obtidos com o crescente número de pesquisas relacionadas com o assunto. Prova disto são os vários estudos realizados em todo mundo pelos mais diversos pesquisadores, tanto, das ciências exatas, jurídicas, sociais e biológicas, formando assim uma união entre tais áreas, aumentando o nível dessas pesquisas com uma interdisciplinaridade, que visa uma melhor compreensão das inter-relações entre os seres vivos e o ambiente em que vivemos (Araújo, 2012).

Autor de correspondência: Roberto Alan Ferreira Araújo, robertoalan.geotech@gmail.com, robertoaraujo@fiponline.edu.br. 
Confalonieri (2001), em sua pesquisa observou que as mudanças climáticas globais afetarão a saúde humana principalmente por meio de alterações nos padrões das doenças infecciosas endêmicas transmitidas pela água (exemplo: cólera, leptospirose) ou por vetores animais (malária, dengue, leishmanioses). Ao serem criadas condições ambientais mais favoráveis à reprodução e à sobrevivência de patógenos e vetores, as mudanças climáticas poderão acelerar os ciclos de transmissão bem como estender as suas áreas de distribuição geográfica, tanto para latitudes quanto para altitudes maiores.

A variabilidade climática é entendida como uma propriedade intrínseca do sistema climático terrestre, responsável por oscilações naturais nos padrões climáticos, observados em nível local, regional e global. Existem diversos fatores climáticos, como a umidade relativa do ar e a temperatura que favorecem a reprodução e sobrevivência de agentes patogênicos no meio ambiente e, principalmente, dos vetores de agente infecciosos, tais como o mosquito Aedes aegypti (Confalonieri, 2003).

As influencias climáticas apresentam interações com a idade do mosquito, por exemplo, a umidade relativa do ar elevada favorece os mosquitos "jovens" e é prejudicial aos "velhos". As temperaturas médias e mínimas influenciam de forma positiva respectivamente a mortalidade dos mosquitos "adultos" e "velhos". Entendendo assim que cidades com temperaturas elevadas favorecem a proliferação e sobrevivência do mosquito (Degallier, 2010).

A Biometeorologia é um dos ramos da meteorologia que trata da influência dos parâmetros atmosférico-meteorológicos sobre o organismo dos seres vivos e no Brasil é pouco explorada até o presente momento. Uma pequena fração de pesquisadores que realizam trabalhos neste ramo da meteorologia e exploram mais a influência sobre as plantas e os animais que sobre os seres humanos (Araújo, 2007).

Problemas da humanidade contemporânea ligados diretamente ao aquecimento global da atmosfera. Apontam a necessidade da previsão dos mesmos como ato inicial, para o qual apontam os avanços tecnológicos atuais e os limites do conhecimento humano para que os princípios de precaução estejam no centro da ideia de prevenção. As condições atmosféricas desempenham forte influência sobre a sociedade e os estados de saúde ou doença do organismo humano se constituem numa das várias manifestações desta interação clima e saúde (Mendonça, 2005).

As condições climáticas da cidade de Fortaleza no estado do Ceará, que está localizado no Norte da região Nordeste, apresentar variabilidade de temperatura do ar ao longo do ano, sendo favoráveis à proliferação do mosquito transmissor das doenças dengue, zika e febre chikungunya, contribuindo para que essas doenças sejam frequentemente verificadas nessa região, afetando seres humanos independente de sua classe social, causando elevados gastos aos cofres públicos e mortalidade em seus casos mais graves (Magalhães e Zanella, 2013). Nessa perspectiva os estudos das relações entre o clima e as enfermidades humanas são de suma importância à saúde pública, haja vista que várias doenças estão relacionadas direta e indiretamente a variabilidade e ritmo climático, como as doenças transmitidas por vetores (Magalhães, 2011).

Desta forma, seguindo essa linha metodológica, diante dos números alarmantes de pessoas doentes, é necessário compreender melhor a influência das variáveis meteorológicas na prevalência das doenças transmitidas pelo mosquito Aedes Aegypti. Assim, diante da perspectiva descrita, surge o seguinte questionamento: os aspectos climáticos influenciam nos casos de doenças transmitidas pelo mosquito Aedes aegypti. Com isso este trabalho avalia a correlação da influência de variáveis meteorológicas com a prevalência das doenças transmitidas pelo mosquito Aedes aegypti do município de Fortaleza, visando obter dados que possam servir para futuras tomadas de decisões por parte dos órgãos responsáveis da área de prevenção e combate ao mosquito bem como da própria defesa civil municipal.

\section{Materiais e Métodos}

Neste capítulo são descritos os materiais e os métodos que foram utilizados na pesquisa, os critérios de seleção da amostra, instrumento de coleta de dados, materiais e técnicas, além de caracterizar o tipo de estudo e caráter da investigação. Será exposta toda a metodologia usada neste estudo, período, amostra, local, análises e procedimentos para com os dados bem como a instrumentação estatística que será executada para fins de obtenção de resposta para as proposições que foram feitas nesta pesquisa.

\subsection{Materiais}

A amostra dos dados das doenças relacionadas ao Aedes Aegypti é oriunda dos Sistemas de Monitoramento Diário de Agravos (SIMDA), da Prefeitura do Município de Fortaleza, que são correlacionados com os dados climáticos do referido Município, oriundos do Instituto Nacional de Meteorologia (INMET) que serão usados como dados de entrada do modelo proposto para obtenção dos resultados.

A pesquisa foi desenvolvida a partir de dados do município de Fortaleza/CE, que atualmente encontra-se dividida em seis Secretarias Executivas Regionais (SER) (Fig. 1). Cada SER possui um setor relacionado à saúde, denominado Coordenadoria Regional de Saúde (CORES).

Optou-se por investigar todas as SER do município escolhido por apresentar elevada prevalência de casos de arboviroses transmitidas pelo mosquito Aedes Aegypti nos últimos 5 anos.

A coleta de dados foi dividida em duas etapas: 1 . Coleta acerca das variações climáticas do município de Fortaleza; 2. Dados sobre a prevalência das arboviroses 


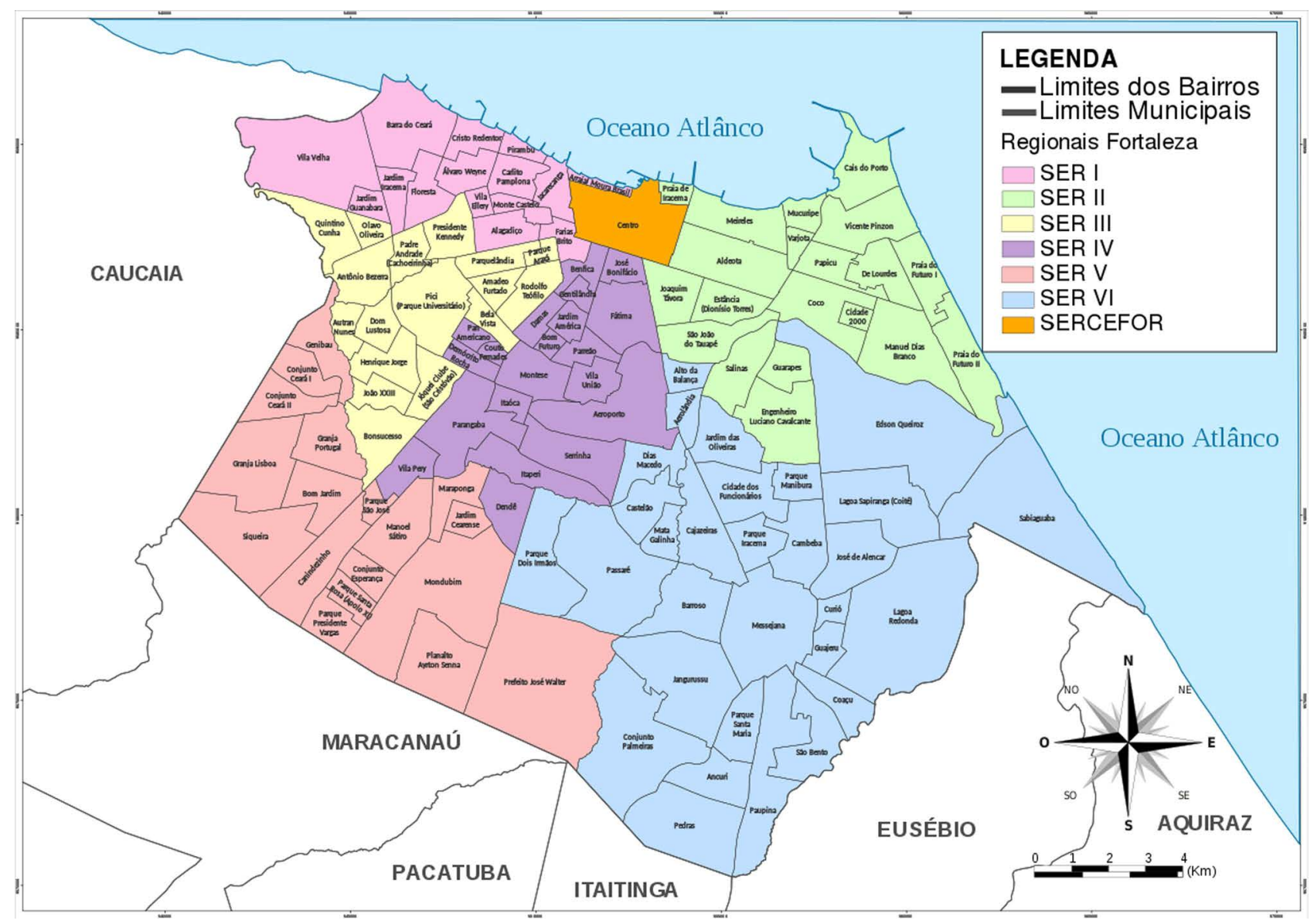

Figura 1 - Mapa de Bairros e divisão por SER. Fortaleza, 2017. Fonte: https://pt.wikipedia.org/wiki/Lista_de_bairros_e_divis\%C3\%B5es_de_Fortaleza.

transmitidas pelo mosquito Aedes Aegypti nos últimos cinco anos; Na primeira etapa os dados foram coletados a partir das informações sobre as variáveis meteorológicas disponibilizadas pelo INMET, em meio eletrônico. Destaca-se que o INMET realiza pesquisas de cada região sobre o clima, o tempo, a agrometeorologia, dentro outros aspectos relacionados à meteorologia.

Para o levantamento das informações acerca da prevalência das arboviroses transmitidas pelo mosquito Aedes Aegypti nos últimos cinco anos, foi consultado o site do SIMDA, o qual é atualizado semanalmente com os dados diários das doenças pesquisadas. Destaca-se que essas doenças são de notificação compulsória, o que garante que os dados coletados retratam a realidade da região pesquisada.

\subsection{Método}

A análise das informações foi realizada sobre a coletânea do material, tendo como objetivo central a compreensão e interpretação. Desta forma, os dados foram expressos através de uma análise descritiva, recorrendo à média, desvio padrão, bem como aos valores de percentuais. Nesta análise estatística foram utilizados os softwares: SPSS V20, Minitab 16 e Excel Office 2010.
Foi definido para este trabalho um nível de significância (quanto admitimos errar nas conclusões estatísticas, ou seja, o erro estatístico que estamos cometendo nas análises) de 0,05 (5\%). Lembramos também que todos os intervalos de confiança construídos ao longo do trabalho, foram construídos com $95 \%$ de confiança estatística.

Foram utilizados testes pertinentes para a análise realizada:

A Correlação de Pearson foi utilizada, essa técnica serve para "medir" (mensurar) o quanto as variáveis estão interligadas, ou seja, a quanto uma está relacionada com a outra. Também utilizada para validar variáveis. Os resultados são dados em percentual, por isso, fica fácil de entender. Vale lembrar que podemos ter valores positivos e negativos.

A metodologia desta técnica segue da seguinte maneira:

$$
\rho_{x y}=\frac{E[X Y]-\mu_{x} \mu_{y}}{\sigma_{x} \sigma_{y}} \operatorname{com}-1 \leq \rho_{x y} \leq 1
$$

em que $E[X Y]=\sum_{i} \sum_{j} x_{i} \cdot y_{j} \cdot \rho\left(x_{i}, y_{i}\right)$. 
Após o calculo das diversas correlações em um único recorte temporal, define-se uma tabela denominada Matriz de Correção.

O Teste de Correlação para testar o coeficiente de correlação é utilizado para testar o coeficiente de correlação entre duas variáveis.

As hipóteses testadas são:

$$
\left\{\begin{array}{l}
H_{o}: \rho=0 \\
H_{1}: \rho \neq 0
\end{array}\right.
$$

Este teste pode ser feito através da variável $t_{0}=\rho \sqrt{\frac{n-2}{1-\rho^{2}}}$ que tem distribuição $t$ de Student com $(n-2)$ graus de liberdade. Para realizar o teste procedem-se como nos demais testes de hipóteses.

Lembramos que o resultado de cada comparação possui uma estatística chamada de p-valor. Esta estatística é que nos ajuda a concluir sobre o teste realizado. Caso esse valor seja maior que o nível de significância adotado (erro ou $\alpha$ ), concluímos, portanto que a $H_{0}$ (a hipótese nula) é a hipótese verdadeira, caso contrário ficamos com $H_{1}$, a hipótese alternativa.

A mediana é uma medida de posição, ela nos divide a amostra ao meio, ou seja, que $50 \%$ dos indivíduos estão acima do valor da mediana e 50\% abaixo. Esta é uma estatística analisada em relação à média, pois quanto mais próximo seu valor em relação à média, mais simétrica será a distribuição e uma distribuição assimétrica, possui uma grande variabilidade com certeza.

A variabilidade é medida pelo desvio padrão. Quanto mais próximo (ou maior) esse valor estiver em relação á média, maior será a variabilidade, o que é ruim, pois assim não teremos uma homogeneidade dos dados, em geral é explicitada pelo Coeficiente de Variação (CV).

O Coeficiente de Variação é um parâmetro estatístico que avalia o quanto a variabilidade representa da média. $\mathrm{O}$ ideal é que este índice seja o mais baixo possível $(<50 \%)$, pois desta forma, teremos uma baixa variabilidade e consequentemente uma homogeneidade dos resultados.

Os valores mínimo e máximo são respectivamente o menor e o maior valor encontrado na amostra, não tem nada a ver com mais ou menos um desvio padrão.

Os quartis são descritivos de posição, ou seja, não são influenciadas por valores extremos (como a média e desvio padrão). $\mathrm{O} 1^{\circ}$ quartil (Q1) nos mostra a distribuição ate $25 \%$ da amostra e o $3^{\circ}$ quartil (Q3) mostra a distribuição até $75 \%$ da amostra.

O intervalo de confiança (IC) ora somado e ora subtraído da média, nos mostra a variação da média segundo uma probabilidade estatística. Também aqui, esses limites não tem nada a ver com o calculo de mais ou menos um desvio padrão em relação a media. Lembrando que o IC é mais confiável, pois temos uma probabilidade estatística associada em seu cálculo.

\section{Resultados e Discussão}

Nesta seção serão apresentados os resultados descritos pela análise estatística dos dados e suas implicações. Inicialmente, apresenta-se uma caracterização geral dos elementos que compõem a pesquisa e, subsequentemente, prossegue-se a análise com a estatística inferencial e a análise gráfica.

A seguir, foi realizada uma análise descritiva da amostra (Tabela 1), para todas as variáveis. Essa análise foi realizada considerando períodos semestrais e também a totalidade da amostra.

Ressalta-se que as médias e medianas para as endemias são maiores no primeiro semestre do que no segundo semestre do ano, indicando maiores ocorrências de Zika, Chikungunya e Dengue no período chuvoso, assim também como para a precipitação, indicando maiores intensidades de precipitação. Para as outras variáveis, como temperaturas máximas e mínimas e umidade relativa do ar, a diferença entre os dois semestres do ano não é evidente, inferindo uma menor variabilidade nessas variáveis ao longo do ano.

As medidas de dispersão, como o desvio padrão e o coeficiente de correlação (CV), também corroboram a explicação do parágrafo acima. No primeiro semestre os valores dessas variáveis são maiores para as doenças e para a Precipitação, e menores no segundo semestre. Em ambos os semestres a variação dos valores das demais variáveis não apresentou grandes alternâncias para o desvio padrão e o $\mathrm{CV}$, indicando menor variabilidade em relação à média.

Pela análise apresentada na Tabela 1, ficou evidente que as doenças possuem alta variabilidade, isso porque o $\mathrm{CV}$ é sempre maior que $50 \%$, demonstrando que os dados são heterogêneos. A variabilidade nos dados dessas doenças era esperada, uma vez que o número de casos se alterna no decorrer das estações, se constituindo em um dos fatos que motivam esse estudo.

As variáveis meteorológicas apresentaram baixíssima variabilidade, a exceção da precipitação, mostrando que são homogêneas. Pode-se dizer, por exemplo, que a média da Precipitação na amostra foi de $106,1 \mathrm{~mm}$ $( \pm 30,9)$. O comportamento das médias pode ser observado graficamente na Fig. 2.

Os gráficos dos Box plot $(\mathrm{a}-\mathrm{h})$ também corroboram as análises da Tabela 1. Observa-se que para as doenças Zika, Chikungunya e Dengue, há uma evidencia de maior variabilidade, ou seja, não-homogeneidade nos dados do primeiro semestre em relação ao segundo semestre.

\subsection{Análise Inferencial}

\subsubsection{Estação climática: verão}

A análise de correlação proposta pelo estudo iniciouse com a verificação da associação das variáveis climáticas e das doenças em análise para o período que 
Tabela 1 - Estatística descritiva da amostra.

\begin{tabular}{|c|c|c|c|c|c|c|c|c|c|c|c|}
\hline & & Média & Mediana & Desvio padrão & $\mathrm{CV}$ & Q1 & Q3 & Min & $\operatorname{Max}$ & $\mathrm{N}$ & $\mathrm{IC}$ \\
\hline \multirow[t]{3}{*}{ Zika } & $1^{\circ} \mathrm{Sem}$ & 365 & 321 & 288 & $79 \%$ & 98 & 569 & 52 & 993 & 12 & 163 \\
\hline & $2^{\circ} \mathrm{Sem}$ & 44 & 19 & 73 & $168 \%$ & 4 & 43 & 0 & 254 & 12 & 41 \\
\hline & Todos & 204 & 69 & 263 & $129 \%$ & 20 & 300 & 0 & 993 & 24 & 105 \\
\hline \multirow[t]{3}{*}{ Chikung. } & $1^{\circ} \mathrm{Sem}$ & 6.572 & 3.647 & 8.603 & $131 \%$ & 594 & 6.724 & 58 & 24.223 & 12 & 4.867 \\
\hline & $2^{\circ} \mathrm{Sem}$ & 873 & 517 & 992 & $114 \%$ & 251 & 1.175 & 0 & 3.339 & 12 & 561 \\
\hline & Todos & 3.722 & 825 & 6.659 & $179 \%$ & 327 & 3.836 & 0 & 24.223 & 24 & 2.664 \\
\hline \multirow[t]{3}{*}{ Dengue } & $1^{\circ} \mathrm{Sem}$ & 3.933 & 2.470 & 4.022 & $102 \%$ & 1.066 & 6.073 & 465 & 15.686 & 30 & 1.439 \\
\hline & $2^{\circ} \mathrm{Sem}$ & 1.086 & 615 & 1.086 & $100 \%$ & 347 & 1.461 & 0 & 4.598 & 30 & 388 \\
\hline & Todos & 2.509 & 1.345 & 3.254 & $130 \%$ & 526 & 2.675 & 0 & 15.686 & 60 & 823 \\
\hline \multirow[t]{3}{*}{ Todas } & $1^{\circ} \mathrm{Sem}$ & 6.708 & 2.611 & 9.012 & $134 \%$ & 1.187 & 7.997 & 465 & 37.844 & 30 & 3.225 \\
\hline & $2^{\circ} \mathrm{Sem}$ & 1.452 & 757 & 1.704 & $117 \%$ & 372 & 1.923 & 0 & 8.191 & 30 & 610 \\
\hline & Todos & 4.080 & 1.619 & 6.955 & $170 \%$ & 586 & 3.172 & 0 & 37.844 & 60 & 1.760 \\
\hline \multirow[t]{3}{*}{ Precip. } & $1^{\circ} \mathrm{Sem}$ & 185,2 & 144,5 & 122,8 & $66 \%$ & 74,8 & 266,4 & 30,3 & 477,9 & 30 & 43,9 \\
\hline & $2^{\circ} \mathrm{Sem}$ & 24,3 & 10,5 & 33,7 & $138 \%$ & 7,5 & 18,0 & 1,1 & 147,5 & 29 & 12,2 \\
\hline & Todos & 106,1 & 66,2 & 121,1 & $114 \%$ & 10,6 & 146,1 & 1,1 & 477,9 & 59 & 30,9 \\
\hline \multirow[t]{3}{*}{ Temp. máxima } & $1^{\circ} \mathrm{Sem}$ & 31,3 & 31,3 & 0,5 & $1 \%$ & 31,0 & 31,5 & 30,3 & 32,3 & 30 & 0,2 \\
\hline & $2^{\circ} \mathrm{Sem}$ & 31,7 & 31,7 & 0,5 & $2 \%$ & 31,6 & 31,9 & 30,4 & 32,6 & 27 & 0,2 \\
\hline & Todos & 31,5 & 31,5 & 0,5 & $2 \%$ & 31,2 & 31,8 & 30,3 & 32,6 & 57 & 0,1 \\
\hline \multirow[t]{3}{*}{ Temp. mínima } & $1^{\circ} \mathrm{Sem}$ & 24,2 & 24,2 & 0,6 & $3 \%$ & 23,6 & 24,5 & 23,1 & 25,7 & 30 & 0,2 \\
\hline & $2^{\circ} \mathrm{Sem}$ & 24,1 & 23,9 & 0,9 & $4 \%$ & 23,7 & 24,7 & 22,3 & 25,6 & 29 & 0,3 \\
\hline & Todos & 24,1 & 24,1 & 0,8 & $3 \%$ & 23,6 & 24,6 & 22,3 & 25,7 & 59 & 0,2 \\
\hline \multirow[t]{3}{*}{ Umid. relativa } & $1^{\circ} \mathrm{Sem}$ & 80,5 & 80,4 & 3,0 & $4 \%$ & 78,3 & 82,4 & 75,7 & 86,6 & 30 & 1,1 \\
\hline & $2^{\circ} \mathrm{Sem}$ & 73,6 & 73,4 & 1,7 & $2 \%$ & 72,5 & 74,2 & 70,8 & 78,3 & 22 & 0,7 \\
\hline & Todos & 77,6 & 77,1 & 4,2 & $5 \%$ & 74,0 & 80,8 & 70,8 & 86,6 & 52 & 1,2 \\
\hline
\end{tabular}

compreende o verão, visto que de janeiro a março é evidenciado o período chuvoso na região estudada com um volume considerável perante os demais meses (Alves e Kayano, 1991). Os dados estão expostos na Tabela 2.

Tem-se que, para os meses do verão, a Tabela 2 mostra que as maiores correlações, que expressam como cada variável climática explicou a variância dos casos das doenças, foram obtidas para a relação entre Dengue e Precipitação $(53,4 \%)$ e para Dengue e umidade relativa do ar $(58,5 \%)$, ambas estatisticamente significantes a $5 \%$. De maneira semelhante, ao analisar as questões climáticas relacionadas a todas as endemias, os coeficientes significativos relacionam as doenças à Precipitação $(52,2 \%) \mathrm{e}$ à umidade relativa do ar $(56,5 \%)$, significativas a $10 \%$ e $5 \%$, respectivamente.

Esse resultado evidencia que há uma forte relação das doenças com a Precipitação e com a umidade relativa do ar, ou seja, verões mais chuvosos, que tendem a serem períodos mais úmidos, são propícios a uma maior evidência de casos dessas doenças em Fortaleza/CE. Monteiro et al. (2009), observou em seu artigo que há uma correlação positiva forte entre incidência da dengue com a precipitação e a temperatura, particularmente no primeiro semestre de cada ano.
Para as temperaturas máximas e mínimas, as correlações negativas, embora não significativas estatisticamente, apresentaram maiores magnitudes para a Zika. As relações da Zika com essas temperaturas inferem que a elevação de temperaturas máximas e diminuição de temperaturas mínimas tendem a reduzir o número de casos dessas endemias na região em estudo. Destaca-se ainda a correlação positiva e expressiva entre Zika e umidade relativa do ar, em que a explicação da variância dos casos ocorridos para essa endemia foi da ordem de $61 \%$, contudo, não apresentou significância estatística.

Para Chikungunya, a umidade relativa do ar tem pouca relação com a evidência de casos. Ou seja, a explicação da sua variabilidade relacionada ao número de casos dessa endemia foi apenas de 8,5\%.

De maneira a promover uma melhor visualização dessas relações, elaborou-se uma síntese das relações mais expressivas entre as doenças e as variáveis meteorológicas, apresentada na Fig. 3.

Por meio da Fig. 3, é possível observar que Zika e Chikungunya possuem relações inversas às temperaturas mínimas e máximas, enquanto que para Dengue e a análise conjunta de todas as endemias, a umidade relativa do ar foi a variável que apresentou maior relação com suas 
a)

Interval Plot of Zika

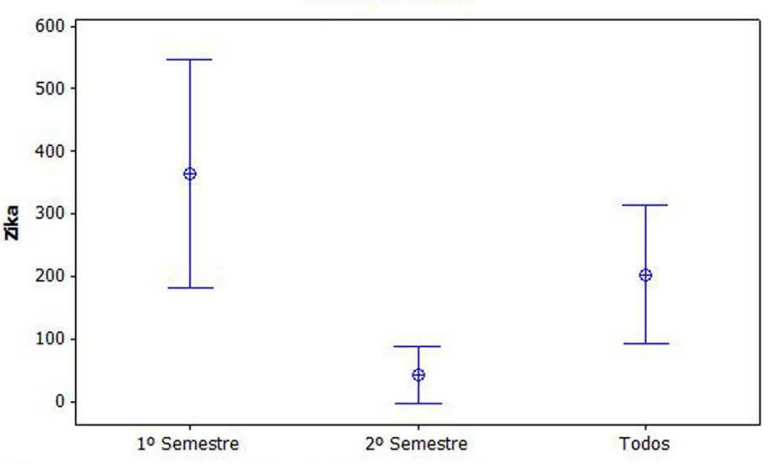

c) Interval Plot of Dengue

$95 \%$ CI for the Mean

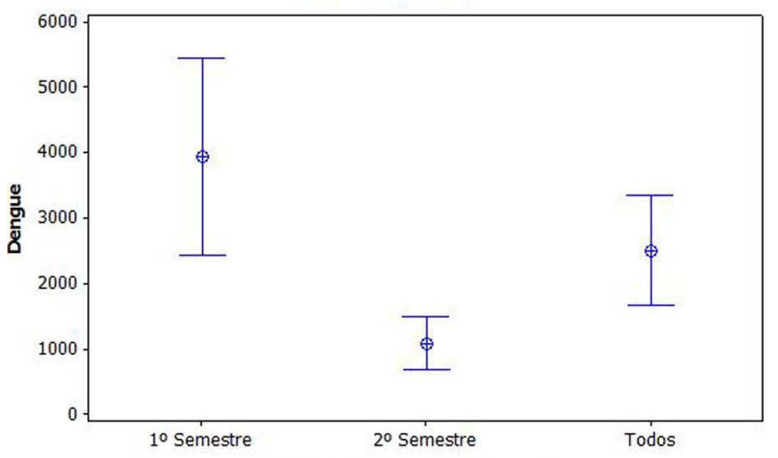

e) Interval Plot of Precipitacao 95\% CI for the Mean

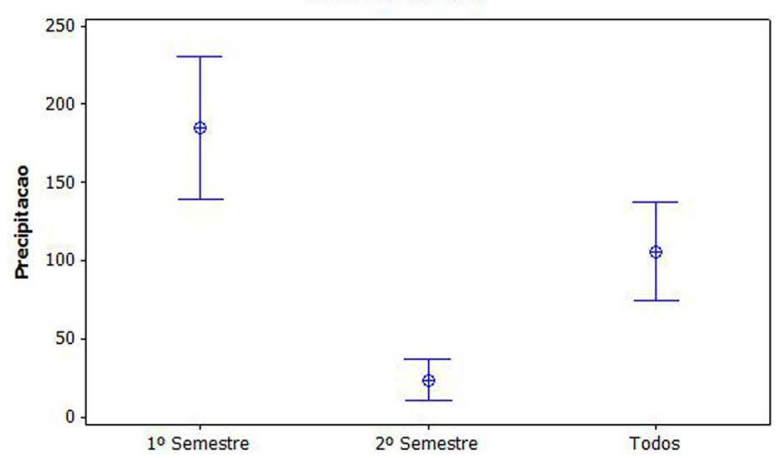

g) Interval Plot of Temp Minima $95 \% \mathrm{CI}$ for the Mean

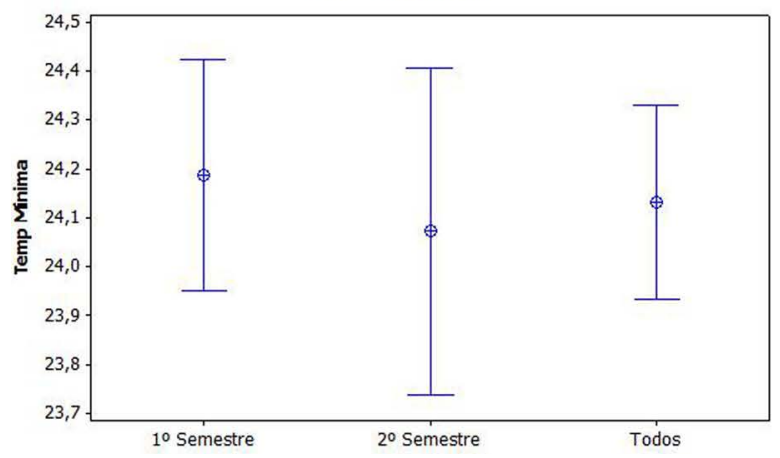

b) Interval Plot of Chikungunya

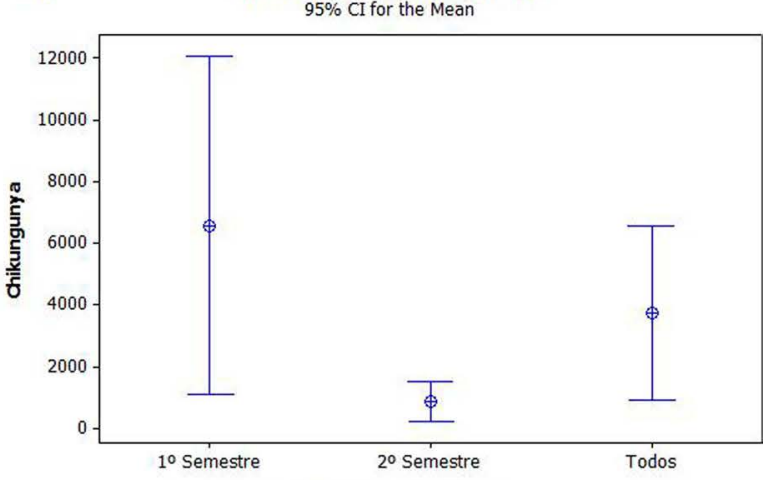

Interval Plot of Todas

d) Interval Plot of Todas

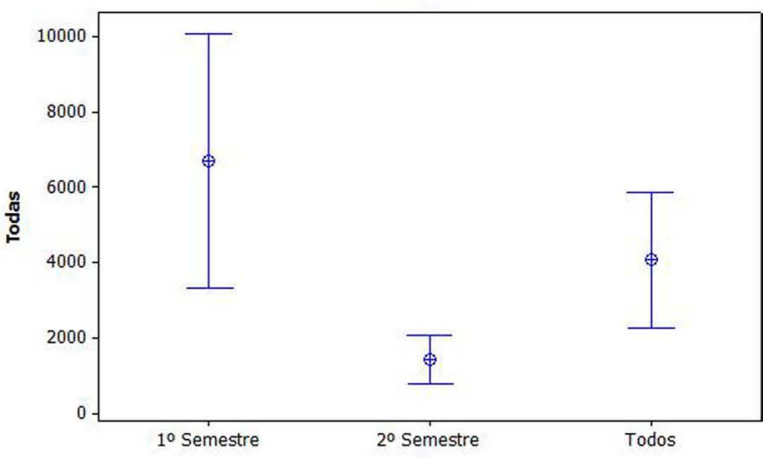

f)

Interval Plot of Temp Maxima $95 \%$ CI for the Mean

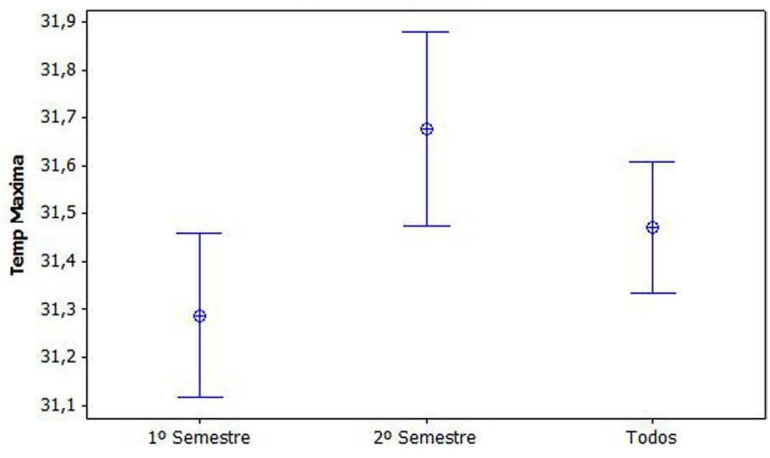

h) Interval Plot of Umidade Relativa $95 \%$ CI for the Mean

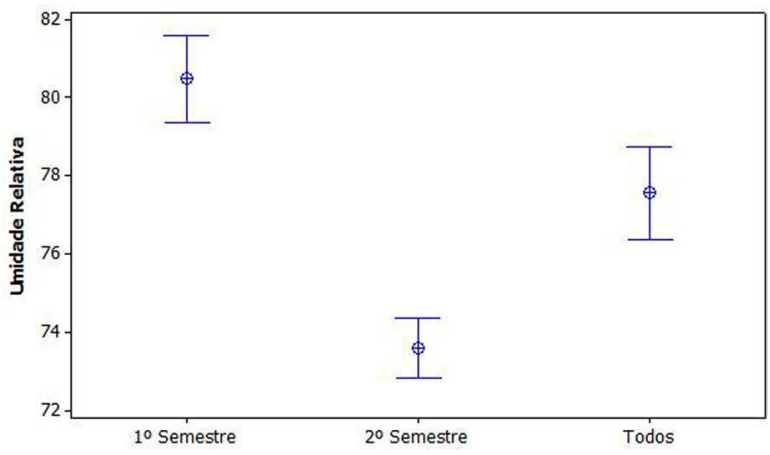

Figura 2 - Blox plots e intervalo de Confiança para: a) Média de "Zika"; b) Média de "Chikungunya"; c) Média de "Dengue"; d) Média de "Todas Patologias"; e) Média de "Preciptação"; f) Média de "Temperatura Máxima"; g) Média de "Temperatura Mínima"; h) Média de "Umidade Relativa do ar". 
Tabela 2 - Correlação de doenças com dados climáticos no verão.

\begin{tabular}{|c|c|c|c|c|c|c|c|c|}
\hline \multirow[t]{2}{*}{ Verão } & \multicolumn{2}{|c|}{ Zika } & \multicolumn{2}{|c|}{ Chikungunya } & \multicolumn{2}{|c|}{ Dengue } & \multicolumn{2}{|c|}{ Todas } \\
\hline & Coef. & P-valor & Coef. & P-valor & Coef. & P-valor & Coef. & P-valor \\
\hline Precipitação & $29,9 \%$ & 0,625 & $1,9 \%$ & 0,976 & $53,8 \%$ & $0,047^{* * *}$ & $52,2 \%$ & $0,056^{* * *}$ \\
\hline Temp. máxima & $-50,7 \%$ & 0,383 & $-26,9 \%$ & 0,662 & $-39,0 \%$ & 0,188 & $-37,9 \%$ & 0,202 \\
\hline Temp. mínima & $-73,6 \%$ & 0,156 & $8,5 \%$ & 0,892 & $-6,2 \%$ & 0,832 & $-4,8 \%$ & 0,871 \\
\hline Umidade relativa & $61,0 \%$ & 0,274 & $8,5 \%$ & 0,892 & $58,5 \%$ & $0,036^{* * *}$ & $56,5 \%$ & $0,044^{* *}$ \\
\hline
\end{tabular}

** Significante a $5 \%$;

${ }^{* * *}$ Significante a $10 \%$.

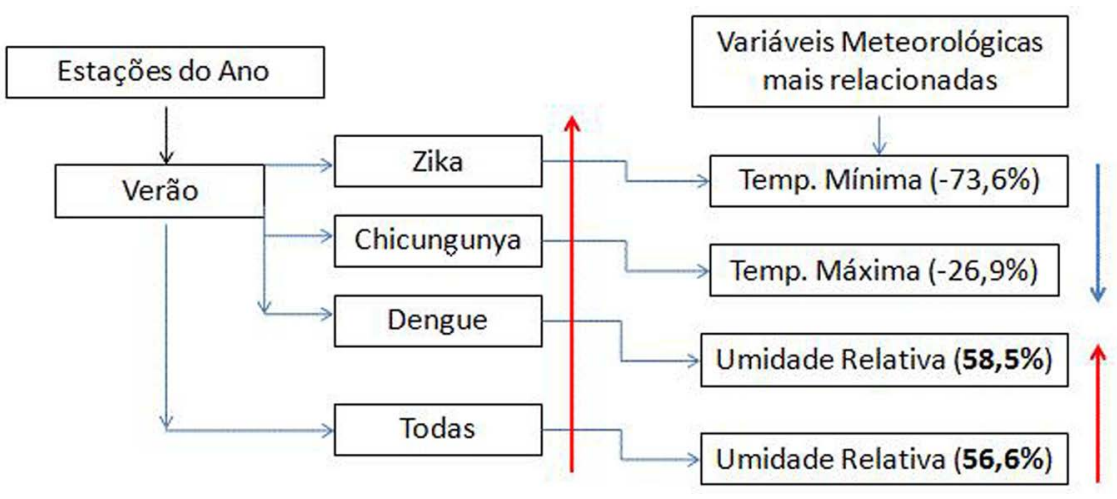

Figura 3 - Relações das endemias com as variáveis meteorológicas no verão.

variabilidades. A umidade relativa do ar foi a variável independente que mais se correlaciona com os casos de dengue mostrando uma influência estatística maior na propagação da dengue entre as variáveis climáticas (Magalhães e Zanella, 2015).

\subsubsection{Estação climática: inverno}

O inverno no hemisfério sul se inicia entre os dias 20 e 21 de junho e se estende até o período entre os dias 22 e 23 de setembro. Em Fortaleza, essa estação corresponde ao fim do período chuvoso e início do período seco. As relações entre as variáveis climáticas e as doenças durante o inverno estão expostas na Tabela 4.

$\mathrm{Na}$ estação de inverno (Tabela 4), estação que marca o fim da quadra chuvosa em fortaleza (fevereiro a maio) e o início de um período mais seco, algumas relações envolvendo as variações de temperaturas máximas e mínimas podem ser destacadas. Inicialmente, a única relação que se apresentou significativa a $5 \%$ foi a que conecta as arboviroses agrupadas com as temperaturas mínimas $(51,3 \%)$. Ou seja, quanto mais intensas são as temperaturas mínimas nesse período, maior a chance de ocorrência dessas doenças. Portanto comprova o que os resultados encontrados neste estudo corroboram com os resultados apresentados por Magalhães e Zanella (2013) afirmando que a precipitação pluviométrica concentra-se no primeiro semestre do ano, com os maiores totais pluviométricos distribuídos entre os meses de fevereiro a maio, período que se verifica um maior número de casos das doenças.

Para as temperaturas mínimas, embora não significativas, a maior correlação foi de $62,8 \%$ quando relacionada à Chikungunya, seguidos de $32,2 \%$ associada à Zika e de $15,9 \%$ relacionada à ocorrência de Dengue. Com relação a temperaturas máximas, as maiores magnitudes estão associadas à ocorrência de Zika $(62,2 \%)$ e para o agregado das doenças (33,3\%). Fuller et al. (2017), levantam a hipótese de que de duas a três semanas após um grande evento de chuva, haverá maior abundância de mosquitos e maior transmissão vetorial de ZIKA.

Tabela 4 - Correlação de doenças com dados climáticos no inverno.

\begin{tabular}{|c|c|c|c|c|c|c|c|c|}
\hline \multirow[t]{2}{*}{ Inverno } & \multicolumn{2}{|c|}{ Zika } & \multicolumn{2}{|c|}{ Chikungunya } & \multicolumn{2}{|c|}{ Dengue } & \multicolumn{2}{|c|}{ Todas } \\
\hline & Coef. & P-valor & Coef. & P-valor & Coef. & P-valor & Coef. & P-valor \\
\hline Precipitação & $-15,1 \%$ & 0,775 & $21,7 \%$ & 0,680 & $11 \%$ & 0,695 & $-7,8 \%$ & 0,782 \\
\hline Tem. max & $62,2 \%$ & 0,144 & $19 \%$ & 0,719 & $-6,2 \%$ & 0,827 & $33,3 \%$ & 0,226 \\
\hline Temp. min & $32,2 \%$ & 0,534 & $62,8 \%$ & 0,181 & $15,9 \%$ & 0,572 & $51,3 \%$ & $0,050 * *$ \\
\hline UR & $28,3 \%$ & 0,645 & $79,5 \%$ & 0,108 & $39,1 \%$ & 0,208 & $25,6 \%$ & 0,422 \\
\hline
\end{tabular}




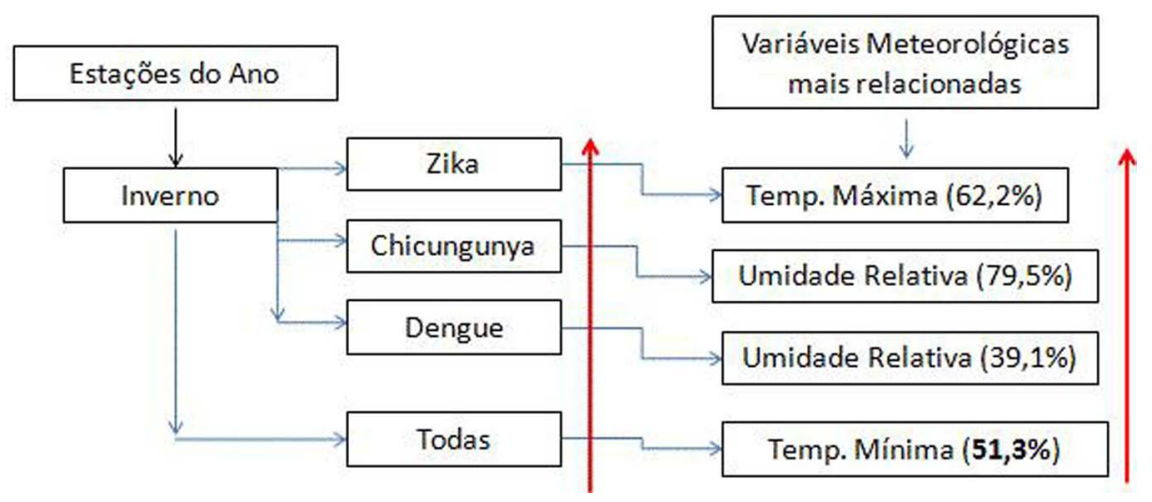

Figura 4 - Relações das endemias com as variáveis meteorológicas no inverno.

Para a umidade relativa do ar, ressalta-se a forte relação linear dessa variável com a Chikungunya, expressa por uma correlação de $79,5 \%(p=0,108)$. Dessa forma, infere-se que a variação da umidade relativa do ar explicou cerca de $80 \%$ dos casos dessa endemia no inverno.

Ademais, a precipitação apresentou baixos valores de correlação entre as variáveis, todos não significativos. Destaca-se ainda que essa variável apresentou relação inversa com a ocorrência de Zika (-15,1\%) e com as endemias agrupadas $(-7,8 \%)$.

De acordo com as correlações observadas, a representação gráfica das mais importantes relações entre as variáveis no inverno está na Fig. 4.

Por meio da Fig. 4, é possível observar a importância da Temperatura Máxima, da Temperatura Mínima e da Umidade Relativa para explicar a ocorrência dessas doenças, assim como a pouca importância da precipitação nessa época do ano, consubstanciando a análise apresentada anteriormente.

\section{Considerações Finais}

De acordo com a abordagem realizada na presente pesquisa, constatou-se que o município de Fortaleza apresenta condições climáticas favoráveis para a proliferação do mosquito Aedes aegipty, bem como para o progresso das doenças Dengue, Chikungunya e Zika.

Diante dessas evidências, imaginamos um cenário não muito confortável em Fortaleza/CE. Os dados aqui apresentados apontam para uma realidade preocupante, pois as variáveis meteorológicas do município contribuem para a proliferação do mosquito Aedes aegypti, elevando assim a prevalência de casos das arboviroses Dengue, Chikungunya e Zika. O seu clima quente na maior parte do ano proporciona maior incidência dessas doenças.

Nessa perspectiva como produto final desta pesquisa será desenvolvido em prol da população uma ação de caráter educativo nas Unidades de Atenção Primária à Saúde (UAPS) do município de Fortaleza, para a conscientização da população sobre a importância dos cuidados a serem tomados para a prevenção das doenças transmitidas pelo mosquito, principalmente, nos períodos do ano em que as variáveis meteorológicas influenciam para maior incidência das doenças.

Devido à complexidade das relações entre fatores climáticos e essas doenças, outros estudos complementares no campo e com outros modelos matemáticos serão necessários para que se possam entender melhor os aspectos que levam ao foco do mosquito Aedes aegypti.

\section{Referências}

ALVES, J.M.B.; EKAYANO, M.T. Estudo preliminar da precipitação no sul do Ceará durante a préestação chuvosa. Boletim Climanálise, v. 6, n. 02, p. 41-50, 1991.

ARAÚJO, R.A.F.; FIRMINO, J.L.N.; GOMES-FILHO, M.F.; DANTAS, R.T. Análise da relação da incidência de Infecção Respiratória Aguda (I.R.A), com as variáveis meteorológicas em Campina Grande. Revista Fafibe On Line, v. 3, n. 3, 2007.

ARAUUJO, R.A.F. Utilização de Modelos Matemáticos/Estocásticos na Avaliação da Ocorrência de Dengue em Função do Clima no Estado da Paraíba. Tese de Doutorado, Departamento de Meteorologia, Universidade Federal de Campina Grande, Campina Grande, 2012.

CONFALONIERI, U.E.C. Variabilidade climática, vulnerabilidade social e saúde no brasil. Terra Livre, v. 1, n. 20, p. 193-204, 2003.

DEGALLIER, N. Impactos climáticos sobre a transmissão da dengue no nordeste do Brasil. Projeto Clima do Atlântico Tropical e Impactos sobre o Nordeste, FUNCEME: Fortaleza, p. 331-337, 2010.

FULLER T.L.; CALVET G.; CALVET, G.; ESTEVAM, C.G.; ANGELO, J.R.; ABIODUN, G.J.; HALAI, U.; DE SANTIS, B.; SEQUEIRA, P.C.; ARAUJO, E.M.; SAMPAIO, S. A.; MENDONÇA, M.C.L.; FABRI, A.; RIBEIRO, R.M.; HARRIGAN, R.; SMITH, T.B.; GABAGLIA, C.R.; BRASIL, P.; FILIPPIS, A.M.B.; NIELSEN-SAINES, K. Behavioral, climatic, and environmental risk factors for Zika and Chikungunya virus infections in Rio de Janeiro, Brazil, 2015-16. PLoS ONE, v. 12, n. 11, e0188002, 2017.

MAGALHÃES, G.B.; ZANELLA, M.E. Comportamento espacial da dengue e sua relação com o clima na região 
metropolitana de fortaleza. Revista Brasileira de Climatologia, v. 12, n. 01, p. 114, 2013.

MAGALHÃES, G.B.; ZANELLA, M.E. A variabilidade climática e a frequência de dengue em Fortaleza, CE, Brasil. Rede - Revista Eletrônica do PRODEMA, v. 9, n. 1, p. 35-50, 2015.

MAGALHÃES, G.B. Chuva e doenças em Fortaleza-ce na perspectiva das mudanças climáticas. In: IV Simpósio Internacional de Climatologia, 2011, João Pessoa. IV
Simpósio Internacional de Climatologia, São Paulo: Sociedade Brasileira de Meteorologia, 2011.

MENDONÇA, F. Clima, tropicalidade e saúde: uma perspectiva a partir da intensificação do aquecimento global. Revista Brasileira de Climatologia, v. 1, n. 1, p. 100-112, 2005.

License information: This is an open-access article distributed under the terms of the Creative Commons Attribution License (type CC-BY), which permits unrestricted use, distribution and reproduction in any medium, provided the original article is properly cited. 